\title{
Delivering Interprofessional Education to Embed Interdisciplinary Collaboration in Effective Nutritional Care
}

\author{
Julie Santy-Tomlinson, Celia V. Laur, and Sumantra Ray
}

\begin{abstract}
Previous and forthcoming chapters describe how to improve nutrition care with an emphasis on interdisciplinary approaches. Developing and improving the skills and knowledge of the interdisciplinary team through interprofessional education are essential for embedding evidence-based, collaborative, nutritional care. This capacity building in turn supports delivery of effective nutritional care for older adults.
\end{abstract}

\section{Keywords}

Interdisciplinary teams · Interprofessional education $\cdot$ Nutritional care

This chapter is a component of Part I: Nutritional Care in Old Age.

For an explanation of the grouping of chapters in this book, please see Chap. 1: 'Overview of Nutrition Care in Geriatrics and Orthogeriatrics'.

\footnotetext{
J. Santy-Tomlinson $(\bowtie)$

Orthopaedic Nursing, Odense University Hospitals/University of Southern Denmark, Odense, Denmark

e-mail: juliesanty@tomlinson15.karoo.co.uk

C. V. Laur

Women's College Hospital Institute for Health System Solutions and Virtual Care, and Women's College Research Institute, Women's College Hospital, Toronto, ON, Canada

NNEdPro Global Centre for Nutrition and Health, Cambridge, UK

e-mail: Celia.Laur@wchospital.ca; cvlaur@uwaterloo.ca

S. Ray

NNEdPro Global Centre for Nutrition and Health, Cambridge, UK

School of Biomedical Sciences, Ulster University at Coleraine, Coleraine, UK

School of Humanities and Social Sciences, University of Cambridge, Cambridge, UK e-mail: S.Ray@nnedpro.org.uk
}

Ó. G. Geirsdóttir, J. J. Bell (eds.), Interdisciplinary Nutritional Management and 


\section{Learning Outcomes}

By the end of this chapter, you will be able to:

- Describe the importance of education in developing effective evidence-based interdisciplinary nutritional care.

- Discuss the benefits of interprofessional education in improving teamworking and collaboration in nutritional care.

- Summarise approaches to interprofessional education for nutritional care.

- Outline the value of education in practice improvement initiatives for nutrition care.

\subsection{Introduction}

Best practice in nutritional care needs to be evidence-based and delivered using a team approach. Even though nutrition specialists such as dietitians are the experts in the management of disease-related malnutrition, it is essential that effective evidence-based nutritional care is delivered by all members of the healthcare team. This need requires a collaborative culture where every practitioner has a fundamental understanding of their own role and the role of others in meeting the nutritional needs of their patients and the public [1]. This collaborative approach will not only improve care for all older adults but will also allow nutrition specialists to direct their expertise and specialist resources where they are most needed, including supporting high-risk individuals.

Healthcare practitioners rarely practise in isolation, working collaboratively with other health and social care professionals to achieve patient-centred goals using the collective skills of the team [2,3]. The importance of interdisciplinary team approaches in the prevention and management of malnutrition in hospitalised older people has been discussed frequently throughout this book (Chaps. 1, 6, and 13). This chapter introduces the education perspectives of developing interdisciplinary teams. For clarity, working definitions of common terms used when referring to interdisciplinary collaboration and interprofessional education are provided in Table 12.1.

\subsection{The Role of Interdisciplinary Teams}

The complexity of human health, combined with the cross-cutting nature of nutrition, means that individuals and communities need all the skills of the healthcare team [5] in all aspects of nutritional care. An example of interdisciplinary nutrition care is monitoring food intake in hospital, where staff involved in supervising and supporting eating and drinking may record food intake, the 
Table 12.1 Working definitions of common terms relating to interdisciplinary/interprofessional collaboration in education

\begin{tabular}{ll}
$\begin{array}{l}\text { Silo (professional } \\
\text { silo) }\end{array}$ & $\begin{array}{l}\text { A culture in which healthcare professionals operate solely within their } \\
\text { own profession and avoid sharing information or collaborating, which } \\
\text { may cause inadvertent duplication of efforts and resources. }\end{array}$ \\
Multidisciplinary & $\begin{array}{l}\text { Professionals from several different disciplines collaborating, with each } \\
\text { drawing on their own professional knowledge and skills applied to a } \\
\text { common focal point. }\end{array}$ \\
\hline Interdisciplinary & $\begin{array}{l}\text { Professionals from several different disciplines collaborating by } \\
\text { integrating and synthesising knowledge and skills from their different } \\
\text { disciplines. Multiple angles on solving shared problems can offer } \\
\text { innovative solutions that cannot be generated by a single discipline } \\
\text { alone. }\end{array}$ \\
Transdisciplinary & $\begin{array}{l}\text { Professional collaboration through creating a new discipline that } \\
\text { transcends the boundaries of disciplinary roles with a polymathic } \\
\text { approach that does not sit neatly in any one discipline. Nutrition can be } \\
\text { considered transdisciplinary in relation to the natural, biomedical and } \\
\text { social sciences as well as the humanities. }\end{array}$ \\
Collaboration & $\begin{array}{l}\text { Cooperatively working together, sharing responsibility for problem- } \\
\text { solving, making decisions and providing patient care in a culture of } \\
\text { mutual trust, respect and collaboration. Requires a clear recognition of } \\
\text { boundaries, areas of overlap and thresholds for seamless referral and } \\
\text { transfer of care. }\end{array}$ \\
\hline Interprofessional \\
IPE occurs when two or more professions learn with, from and about \\
each other to improve collaboration and the quality of care [4].
\end{tabular}

specialist nutrition practitioner can follow up if intake is low, and a physician can consider low intake as part of their assessment. Each team member has an important role in ensuring that the food intake of a patient is documented and that appropriate action is taken if intake is inadequate, thus positively impacting on patient recovery and care outcomes.

If nutrition care practices are to be improved so that every malnourished or atrisk patient is identified and treated effectively, it is important to identify the barriers impacting the provision of nutrition care [6]. Two of these barriers are; (1) failures in multidisciplinary team collaboration and, (2) gaps in the interdisciplinary education of healthcare professionals.

Effective interdisciplinary teamworking requires team collaboration and involves open communication and information sharing within the team, ensuring that all team members are working towards the same goals. Successful teams work in an environment in which every member of the team is valued and respected and each has a clear role and understanding of the tasks which are their responsibility. These teams are also well-led, well-managed and effectively resourced in an environment in which performance is constantly reviewed and improved with a focus on patient outcomes [7]. An example of a successful practical strategy for the improvement of teamwork and communication can be found in Box 12.1. 


\section{Box 12.1 An example of a successful strategy for improving teamwork}

Communication failures in healthcare teams are associated with medical errors and negative health outcomes. For this reason, team-based communication strategies have increased in the training of future health professionals. For example, in 2018, Brock et al., [8] explored the impact of a simulationbased interprofessional TeamSTEPPS (Team Strategies and Tools to Enhance Performance and Patient Safety, https://www.ahrq.gov/teamstepps/index. html) training programme on student attitudes, knowledge and skills around interprofessional communication. In this study, medical, nursing, pharmacy and physician assistant students $(n=306)$ took part in a 4-h training that included a 1-h TeamSTEPPS didactic session and three 1-h team simulation and feedback sessions. Students worked in groups balanced by a professional programme in a self-selected focal area. Pre-and post-assessments found differences in attitudes towards team communication $(p<0.001)$, motivation $(p<0.001)$, utility of training $(p<0.001)$ and self-efficacy $(p=0.005)$. Attitudinal shifts for TeamSTEPPS skills included team structure $(p=0.002)$, situation monitoring $(p<0.001)$, mutual support $(p=0.003)$ and communication $(p=0.002)$. Shifts were reported for knowledge of TeamSTEPPS $(p<0.001)$, advocating for patients $(p<0.001)$ and communicating in interprofessional teams $(p<0.001)$. This is just one example of a training programme used to support team communication strategies for trainees.

How teams 'work together' may be considered as a continuum progressing from independently working in silos through multidisciplinary, interdisciplinary and then transdisciplinary approaches (Table 12.1; Chap. 1, Fig. 1.1). Progression along this continuum is contingent on collaboration, mutual respect, trust and shared responsibilities and decision-making. However, these qualities alone are inadequate to support models and teams that embrace multi-, inter- or transdisciplinary approaches. Sharing of knowledge and skills should be considered a cornerstone of advancing teams working together towards realising transdisciplinary care.

\subsection{Nutrition Education and Interdisciplinary Team Collaboration}

The education of health professionals aims to ensure that all clinical decisions are supported by accurate and current information that reflects the best available evidence to achieve the best patient outcomes [9]. Unfortunately, health professionals are often not well informed about best practice in nutritional support, and their education is central to successfully implementing team approaches to nutritional assessment and interventions $[10,11]$. The specific aim of interprofessional nutrition education for health professionals is to ensure that clinicians delivering healthcare to older people who are, for example, malnourished or at risk of malnutrition, 
have the right skills, knowledge and attitudes to deliver evidence-based interdisciplinary care. This approach must extend not only across disciplines but also across settings and the care continuum to effectively manage and prevent malnutrition across communities and healthcare settings. To achieve this, nutrition education is such an important foundation for fundamental healthcare practice that it needs to be embedded in the curricula of undergraduate, postgraduate and continuing education for all healthcare professionals, no matter which discipline [12, 13]. Interprofessional nutritional education has significant potential to be both a facilitator of evidencebased nutritional care through skills enhancement and a driver of better interdisciplinary team collaboration, which then leads to beneficial skill sharing.

It has been recognised for many years that health professionals need education and training to support best practice in nutritional care, but the literature suggests that this goal has not been achieved $[14,15]$. The evidence for a lack of nutritional education for medical students is particularly strong [12]. However, even though much of the literature in this area considers medical education, this is also true of most other health professional disciplines, creating a professional culture in which nutritional care is sometimes seen as 'someone else's job'.

Considering the transdisciplinarity of nutritional care and the benefits of interdisciplinary team collaboration, it is clear these need to be considered together. However, effective collaboration remains relatively rare in most healthcare settings [16]. One explanation for this is that practitioners from different professions tend to work in 'silos', groups in which one health professional group is educated and practises together but in isolation from others [17, 18]. For these barriers to be overcome, it is widely proposed that education for all healthcare professionals needs to be interprofessional $[19,20]$ focusing on knowledge, skills and attitudes, along with the critical appraisal skills to identify appropriate evidence [21, 22]. This could be as simple as, for example, having dietitians teach medical students or support development of course material to integrate nutrition throughout the curriculum.

Cultural reform that enables students to develop at least some of the skills of other professions, as well as their own, is recommended for collaboration to be realised [23]. While most health professionals receive fundamental education and training about nutritional aspects, this is rarely sufficient for them to be confident and competent in providing effective nutritional care, especially in settings where nutritionists and dietitians are scarce or not available [1]. Interprofessional education is proposed as one of the ways of bridging skills gaps across the interdisciplinary team. In the case of nutritional care, it is one way that other healthcare professionals can learn about and operationalise the role of the nutritionist and their own and each other's roles in preventing and managing malnutrition.

Interdisciplinary healthcare is essential for the person-centred and effectively coordinated complex care required for frail older people. When healthcare is not person centred and/or not coordinated across the continuum, it is less effective and more likely to have a detrimental effect on outcomes. Considering this context within the aim of IPE, care for older adults can be improved through an interactive learning process that includes collaboration in itself. In this way, healthcare professionals learn, both individually and in teams, how to collaborate and communicate 
Box 12.2 Case example of improvement in collaborative nutrition care through applied education and practice

In 2014, junior doctors from three hospitals across England were provided training in the basics of nutrition care along with an introduction to change management and leadership. These skills set them up to be nutrition champions, and when the trainees returned to their hospital, they put those skills into practice by running nutrition awareness weeks. To run these weeks, the champions worked with clinicians, administrators, staff and leaders across the hospital (transdisciplinary team building) to highlight the importance of nutrition (nutrition knowledge) and champion an event in their hospital (leadership) [26]. More recently, this model has been expanded on in nutrition education training across the $U K$.

within an interdisciplinary context so that such learning can then be transferred to day-to-day practice [24].

There is limited research that demonstrates the long-term outcomes of IPE on aspects of practice such as performance and care quality, but there are some examples of good practice, and studies that evaluate the impact of education on professional behaviour are challenging to conduct [25]. Even so, health professional educators agree that interprofessional education has significant potential to improve collaborative practice between each of the healthcare disciplines and, hence, have a positive impact on the quality of care [17]. Nutrition education is proposed as an approach to enabling practitioners to understand each other's roles and collaborate more effectively since nutrition is an aspect of practice where there is an important role for every member of the team. An example of such an approach is described in Box 12.2.

\subsection{Approaches to Interprofessional Education for Nutritional Care}

In recognition that education programmes for healthcare professionals inadequately prepare clinicians for a collaborative approach to nutritional care, a coordinated approach to interprofessional education aimed at improving nutrition practice across the entire team is needed. This means that each profession needs to review its current nutrition-related competence and use this to redevelop education curricula and delivery to ensure they facilitate collaborative nutritional practice rather than the current profession-specific programmes [11]. This requires nationwide, possibly global, overhaul of health professional curricula.

\subsubsection{Curriculum}

Curricula are blueprints for the content and delivery of education programmes that describe how learning needs are to be met, how content is to be learned and 
delivered, how the resources and assessment methods are to be and how effectiveness of programmes is to be evaluated [27]. The development of curricula needs to be driven by the competencies needed by the whole multidisciplinary team to deliver effective nutritional care led by dietetic practitioners [28]. For this to happen, those designing IPE programmes need to define common learning outcomes for all leaners who will be involved in the IPE programme [17]. These learning outcomes need to reflect the core collective nutrition learning needs [15] as well as the individual learning programmes in which students are individually engaged and require development through collaborative approaches that involve faculty from all the health professions involved. Such coordination is not a simple undertaking and constitutes a common barrier to the development of IPE outcomes that can only be overcome by collaborative working in education/academic institutions.

An essential aspect of curriculum is the design of assessment that will enable students to demonstrate learning that reflects the learning outcomes of the programme. The strategy for assessment will need to ensure that students can demonstrate the skills, competencies, knowledge and attitudes needed for successful collaborative nutritional care.

\subsubsection{Delivery}

It is important to consider how healthcare students and practitioners learn to work together across disciplines [29]. The way in which curricula are delivered needs to meet the learning needs of all health professionals being educated at the same time as bringing them together to learn. This involves careful consideration of teaching and learning methods to be employed as these need not only to meet the learning outcomes but fit the different needs of all team members. As clinical experts, dietetic practitioners have a central role in educating other health professionals for best practice in nutrition care in such programmes [29]. At a time when the value of traditional learning methods such as face-to-face lectures and seminars is being questioned, innovative and imaginative methods such as team-based learning, inquiry-based learning, evidence-based learning, scenario-based learning and problem-based learning are increasingly being used to foster collaboration in interprofessional healthcare education. A common theme in all these options is that students from varied professional groups use clinical exemplars to work together to understand the needs of patients and collaborate in identifying priorities, plan and deliver care.

Team-based learning (TBL) has, for example, been used successfully in providing interprofessional education relating to nutrition and lifestyle interventions [30, 31] but is less tried and tested in the field of practice improvement in preventing and managing malnutrition. In other fields of healthcare, TBL has been shown to engage students in authentic experiences of working in teams to solve real-life clinical problems [29]. Students work in teams and follow a specific sequence of activities throughout a unit of learning involving three phases [32]: (1) a preparation assignment prior to the first session, (2) individual and group readiness assurance tests and, (3) group activity that requires students to apply the material from the 
preparation assignment to a 'real-world' scenario. Using modes of learning such a TBL that engage students collaboratively in important patient safety issues such as malnutrition has the potential to significantly influence practice in the future.

There has been much discussion, especially since the arrival of the COVID-19 pandemic, about the value of using online methods to support learning. Many education programmes have successfully used 'blended learning' approaches, using both online and small group face-to-face sessions. It has been suggested that this may be a way to overcome some of the traditional barriers to interprofessional education discussed earlier [33]. Blended learning is an approach to teaching and learning that combines aspects of face-to-face and online interactions using appropriate technology and the provision of learning materials and opportunities through online platforms. Fundamental elements of this flexible approach are that different learning styles are accommodated and learners actively participate in learning with a focus on peer-learning and self-reflection [34]. Both online and face-to-face sessions provide a more rounded learning experience and enable collaborative learning that supports future interprofessional practice.

Few IPE programmes/curricula have been fully described or evaluated using robust methods. It is essential that IPE programmes are fully evaluated to ascertain the success of programmes in delivering the aims of interprofessional collaboration in improving nutrition care. This should not only include learner reactions to the programme and its components but evaluate: (1) whether students' attitudes, perceptions, knowledge and skills have been modified, (2) whether changes in behaviour have occurred and, (3) whether there have been changes in practice across healthcare organisations that lead to benefit to patients [25].

Strategies are needed for the development of an evidence-base for interprofessional nutrition education. There is a need for studies that assess the effectiveness of IPE interventions compared to separate, profession-specific interventions, as well as those which examine the processes relating to the IPE and consequent nutrition practice changes as well as cost-benefit analyses [16].

\subsection{Interdisciplinary Collaboration for Nutritional Care in Action}

Education is an essential aspect of nutrition practice improvement, but it is not sufficient on its own to drive and sustain best practice. Practice improvement initiatives, which include education as part of the strategy, need to be planned, led and evaluated to reflect quality improvement principles.

One example of an organisation which incorporates nutrition education with an interdisciplinary approach to improve nutrition outcomes is the NNEdPro Global Centre for Nutrition and Health (https://www.nnedpro.org.uk/). The NNEdPro Global Centre for Nutrition and Health provides a strategic global approach to advance and implement interdisciplinary collaboration in nutrition knowledge and skills to improve health and well-being. As an international, interdisciplinary think tank, they bring together nutrition education, research, policy and innovation to develop adaptable and 
scalable educational models for nutrition capacity building in health systems (https:// www.nnedpro.org.uk/about-us). Their mission is to develop a critical mass of selfsustaining knowledge, skills and capacity in nutrition and health within the global healthcare and public health workforce, resulting in significantly improved health practices and outcomes. Working with these types of global organisations not only encourages the integration of interdisciplinary nutrition education into medical curricula but also drives forward the importance of applying education to practice to support improved patient and public nutrition care outcomes, including for older adults.

\subsection{Summary}

Education of health professionals is central to developing effective evidence-based interdisciplinary nutritional care, and there are several examples of good practice. Interprofessional education is widely considered an effective approach to improving teamworking and collaboration and needs to be embedded in health professional education curricula using a variety of teaching and learning models and methods.

\section{Take-Home Points}

- To improve nutritional care several barriers need to be overcome including failures in multidisciplinary team collaboration and gaps in the interdisciplinary education of healthcare professionals.

- The aim of interprofessional nutritional education is to ensure that clinicians have the skills, knowledge and attitudes to deliver evidence-based interdisciplinary care to effectively manage and prevent malnutrition.

- Interprofessional nutrition education needs to be embedded in the curricula driven by the competencies needed by the whole multidisciplinary team and common learning outcomes for all leaners reflecting the core collective nutrition learning needs.

- Practice improvement initiatives are needed which include education as part of the strategy, and these need to be evaluated to ascertain the impact on practice.

- An organisational culture that is constructive and open to growth and development is key in enabling cross-disciplinary knowledge sharing from upstream domains considering food production, supply and sustainability through dietary choices, guided interventions and downstream impacts on nutritional status as well as health and disease outcomes in healthcare settings.

\section{References}

1. Bell JJ et al (2021) Nutritional care of the older patient with fragility fracture: opportunities for systematised, interdisciplinary approaches across acute care, rehabilitation and secondary prevention settings. In: Falaschi P, Marsh D (eds) Orthogeriatrics: the management of older patients with fragility fractures. Springer, Cham, pp 311-329 
2. Dinh JV et al (2021) The study of teamwork processes within the dynamic domains of healthcare: a systematic and taxonomic review. Front Commun 6:3

3. Wei $\mathrm{H}$ et al (2020) A culture of caring: the essence of healthcare interprofessional collaboration. J Interprof Care 34(3):324-331

4. CAIPE (2002) Interprofessional education: today, yesterday and tomorrow. The Centre for the Advancement of Interprofessional Education 2002 [cited 2021 28.04.2021]. https://www.caipe.org/resources/publications/caipe-publications/ caipe-2002-interprofessional-education-today-yesterday-tomorrow-barr-h

5. Thistlethwaite J, Jackson A, Moran M (2013) Interprofessional collaborative practice: a deconstruction. J Interprof Care 27(1):50-56

6. Tappenden KA et al (2013) Critical role of nutrition in improving quality of care: an interdisciplinary call to action to address adult hospital malnutrition. JPEN J Parenter Enteral Nutr 37(4):482-497

7. Margaret Slusser LG, Reed C-R, McGinnis PQ (2018) Foundations of interprofessional collaborative practice in health care, 1st edn. Mosby, Maryland Heights, p 280

8. Brock D et al (2013) Interprofessional education in team communication: working together to improve patient safety. BMJ Qual Saf 22(5):414-423

9. Lehane $\mathrm{E}$ et al (2019) Evidence-based practice education for healthcare professions: an expert view. BMJ Evid Based Med 24(3):103-108

10. Nightingale $J$ (2010) Nutrition support teams: how they work, are set up and maintained. Frontline Gastroenterol 1(3):171-177

11. DiMaria-Ghalili RA et al (2014) Challenges and opportunities for nutrition education and training in the health care professions: intraprofessional and interprofessional call to action. Am J Clin Nutr 99(5 Suppl):1184s-1193s

12. Crowley J, Ball L, Hiddink GJ (2019) Nutrition in medical education: a systematic review. Lancet Planet Health 3(9):e379-e389

13. Ellis G, Sevdalis N (2019) Understanding and improving multidisciplinary team working in geriatric medicine. Age Ageing 48(4):498-505

14. Kohlmeier M et al (2015) Nutrition education for the health care professions. J Biomed Educ 2015:380917

15. Kris-Etherton PM et al (2014) The need to advance nutrition education in the training of health care professionals and recommended research to evaluate implementation and effectiveness. Am J Clin Nutr 99(5 Suppl):1153s-1166s

16. Reeves $S$ et al (2017) Interprofessional collaboration to improve professional practice and healthcare outcomes. Cochrane Database Syst Rev 6(6):CD000072

17. Thistlethwaite JE (2015) Interprofessional education: implications and development for medical education. Educ Med 16(1):68-73

18. Abu-Rish E et al (2012) Current trends in interprofessional education of health sciences students: a literature review. J Interprof Care 26(6):444-451

19. Mladenovic J (2017) Strategies for overcoming barriers to IPE at a health sciences university. J Interprofessional Educ Pract 8:10-13

20. Homeyer $\mathrm{S}$ et al (2018) Effects of interprofessional education for medical and nursing students: enablers, barriers and expectations for optimizing future interprofessional collaboration-a qualitative study. BMC Nurs 17:13

21. Green BN, Johnson CD (2015) Interprofessional collaboration in research, education, and clinical practice: working together for a better future. J Chiropr Educ 29(1):1-10

22. Bridges DR et al (2011) Interprofessional collaboration: three best practice models of interprofessional education. Med Educ Online 16

23. Grace S (2020) Models of interprofessional education for healthcare students: a scoping review. J Interprof Care:1-13

24. Buring SM et al (2009) Interprofessional education: definitions, student competencies, and guidelines for implementation. Am J Pharm Educ 73(4):59

25. Anderson E, Smith R, Hammick M (2016) Evaluating an interprofessional education curriculum: a theory-informed approach. Med Teach 38(4):385-394 
26. Ray S et al (2014) Nutrition education and leadership for improved clinical outcomes: training and supporting junior doctors to run 'Nutrition Awareness Weeks' in three NHS hospitals across England. BMC Med Educ 14:109

27. Lee A, Steketee C, Rogers G, Moran M (2013) Towards a theoretical framework for curriculum development in health professional education. Focus Health Profess Educ 14(3):64-77

28. Hark LA, Deen D (2017) Position of the Academy of Nutrition and Dietetics: interprofessional education in nutrition as an essential component of medical education. J Acad Nutr Diet 117(7):1104-1113

29. Burgess A et al (2020) Interprofessional team-based learning (TBL): how do students engage? BMC Med Educ 20(1):118

30. Pogge E (2013) A team-based learning course on nutrition and lifestyle modification. Am J Pharm Educ 77(5): 103

31. Khalafalla FG et al (2020) Enhancing nutrition and lifestyle education for healthcare professional students through an interprofessional, team-based training program. Curr Pharm Teach Learn 12(12): 1484-1490

32. Reimschisel $\mathrm{T}$ et al (2017) A systematic review of the published literature on team-based learning in health professions education. Med Teach 39(12):1227-1237

33. Chen AK et al (2017) Teaching interprofessional collaborative care skills using a blended learning approach. J Interprofess Educ Pract 8:86-90

34. Swaminathan N et al (2020) Blended learning and health professional education: Protocol for a mixed-method systematic review. J Educ Health Promot 9:46

\section{Recommended Reading}

Crowley J, Ball L, Hiddink G (2019) Nutrition in medical education: a systematic review. Lancet Planet Health 3(9):e379-e389. https://doi.org/10.1016/S2542-5196(19)30171-8

Ray S, Laur C, Douglas P et al (2014) Nutrition education and leadership for improved clinical outcomes: training and supporting junior doctors to run 'Nutrition Awareness Weeks' in three NHS hospitals across England. BMC Med Educ 14:109. https://doi.org/10.1186/1472-6920-14-109

Open Access This chapter is licensed under the terms of the Creative Commons Attribution 4.0 International License (http://creativecommons.org/licenses/by/4.0/), which permits use, sharing, adaptation, distribution and reproduction in any medium or format, as long as you give appropriate credit to the original author(s) and the source, provide a link to the Creative Commons license and indicate if changes were made.

The images or other third party material in this chapter are included in the chapter's Creative Commons license, unless indicated otherwise in a credit line to the material. If material is not included in the chapter's Creative Commons license and your intended use is not permitted by statutory regulation or exceeds the permitted use, you will need to obtain permission directly from the copyright holder. 